\section{LA ORGANIZACIÓN Y BÚSQUEDA DE CONOCIMIENTO EN ENTORNOS AMPLIOS, DISPERSOS PERO LIMITADOS. EL MAPA DE AYUDAS A LA I+D+i DEL CDTI}

\author{
Javier Ponce Martínez \\ Jefe del departamento de promoción de la innovación del Centro para \\ el Desarrollo Tecnológico Industrial
}

\section{THE ORGANIZATION AND SEARCH OF KNOWLEDGE IN WIDE, DISPERSED BUT LIMITED ENVIRONMENTS. RESEARCH MAP ON RTD FROM CDTI}

\begin{abstract}
The arrangement and the search of knowledge within open environments, dispersed but within limits and whose featured information is somewhat structured and contrasted, does not require large and expensive search engines based on endless index files. New light-weight knowledge management systems based upon the use of folksonomies, whose design also caters for better visualization to the uses, provide fast access to the information and potentially the arrangement of results according to the orientation or point of view required by the user. We at the department for the Promotion of Innovation of CDTI have recently launched a such system, through which to provide electronic information services on all of the public aids aimed at business $R \& D$ available from local, autonomous communities and central state governments as well as from the European Union.
\end{abstract}

KEY WORDS: Searching; dispersed and limites environments; categorization; labels; folksonomy; structured knowledge; public incentives; transfers of knowledge.

Mucho se ha escrito y debatido sobre el desarrollo de los sistemas de búsqueda en Internet y su capacidad para atraer a millones de usuarios que los utilizan a diario, y suponen una herramienta hoy imprescindible en buen número de casos. Sin embargo, nada parecido ha ocurrido con aquellos contenidos que, suponiendo un entorno amplio, generalmente disperso pero limitado y de información perecedera, ofrecen una información concreta, relativamente estructurada y contrastada. Cuando estos entornos deben ser difundidos de forma amplia ofreciendo una información precisa en cada búsqueda se requiere la puesta en marcha de herramientas de búsqueda cuyo uso sea de fácil manejo y capaces de ofrecer resultados de elevada exactitud.
RESUMEN: La organización y búsqueda de conocimiento dentro de entornos amplios, dispersos pero limitados y con una información relativamente estructurada y contrastada no necesita de grandes y costosas herramientas de búsqueda con ingentes volúmenes de información indexada. La utilización de ligeros sistemas de gestión del conocimiento haciendo uso de folksonomias y concebidos bajo una buena estrategia de visualización permiten acceder a la información de forma rápida, y con la potencialidad de estructurar el resultado de búsqueda con la orientación o punto de vista deseado por el usuario. Desde el departamento de promoción de la innovación del CDTI hemos puesto en marcha recientemente un sistema de esta naturaleza, a través del cual se presta un servicio electrónico de información sobre el conjunto de ayudas públicas a la I+D+i empresarial que ofrecen las distintas administraciones locales, autonómicas, estatal y europea.

PALABRAS CLAVE: Búsqueda; entornos dispersos y limitados; categorización; etiquetas; folksonomía; estructuración de conocimiento; ayudas públicas; transferencia de conocimiento.

Entornos como los mencionados antes se producen en muchos órdenes de la vida, pero de manera especial en actuaciones donde varias administraciones públicas proyectan acciones hacia el ciudadano. Éste puede ser el caso de España, donde una actuación puede venir influenciada por competencias de la Administración local, autonómica, estatal o incluso la europea dada nuestra pertenencia a la Unión Europea. Si a lo anterior se suma el hecho de estar la acción que quiere realizar la persona física o jurídica condicionada por un alto coste de oportunidad, encontramos un contexto casi perfecto donde se necesitan herramientas que garanticen la precisión y exhaustividad en las búsquedas sobre repositorios amplios pero limitados y relativamente estructurados. 
El departamento de promoción de la innovación del Centro para el Desarrollo Tecnológico Industrial (CDTI) se responsabiliza de las áreas de asesoramiento, del servicio de información oficial, de la relación con los medios de comunicación, de la logística e imagen y de mercadotecnia del organismo. Por tanto, gestiona y participa en las acciones de comunicación y promoción general, presta atención a empresas y emprendedores, coordina las actividades relacionadas con medios, atiende las solicitudes de información recibidas, elabora y edita publicaciones corporativas e informes y apoya en la identificación de sectores, áreas tecnológicas y potenciales empresas beneficiarias de las ayudas del Centro. En concreto, en el área de asesoramiento coordina y gestiona la Red nacional de puntos de información sobre investigación, desarrollo tecnológico e innovación cuya misión es la de informar y asesorar a empresas y emprendedores sobre los instrumentos de financiación pública a la $\mathrm{I}+\mathrm{D}+\mathrm{i}$ que mejor se adapten a sus necesidades independientemente del ámbito geográfico de la ayuda (local, autonómico, estatal o internacional).

En el departamento de promoción de la innovación llevábamos años interesados en la elaboración de un gran repositorio de ayudas públicas a la $I+D+i$ que es una de las materias donde las cuatro administraciones antes mencionadas ofrecen oportunidades de financiación a actuaciones empresariales y de emprendeduría. Fue a finales de 2005 cuando nos planteamos la puesta en marcha de un buscador de acceso libre que fuera capaz de ofrecer información sobre la práctica totalidad de ayudas públicas a la I+D+i. La búsqueda de las ayudas que más se ajustan a las necesidades de cada empresa en cada momento y la preparación de las propuestas de solicitud supone un esfuerzo que en el caso de pequeñas y medianas empresas puede llegar a ser significativo, en función del volumen de la empresa, y dificultar su participación en los programas de ayuda a la I+D.

Estos sistemas de búsqueda abierta pero a la vez precisa en entornos amplios, dispersos pero finitos comparten algunas exigencias con los grandes buscadores de la red para ser viables a largo plazo, como son la necesidad de incorporar nuevos contenidos al repositorio de búsqueda de la manera más rápida y automatizada posible y la conveniencia de ofrecer resultados no nulos. Estas dos exigencias se convierten en fortalezas del servicio cuando se ofrece desde las administraciones: la actualización casi automatizada del contenido del repositorio evita la dedicación de mano de obra abaratando considerablemente el resultado sin perder por ello precisión, y el resultado no nulo en las búsquedas transmite lo amplitud de la actuación ofreciendo posibilidades a prácticamente casi cualquier actuación o iniciativa privada amplificando la actuación de las administraciones.

Desde el departamento de promoción de la innovación conocíamos los desarrollos que se estaban llevando a cabo en el entorno del Laboratorio de Innovación en Tecnologías de la Información (LITI) de la Universidad Politécnica de Madrid sobre contenidos de e-learning. Esto era así dado que, ya en el año 1999, colaboramos conjuntamente con ese laboratorio para la distribución electrónica de la documentación oficial de la presidencia española del programa internacional Eureka, a través de una aplicación que permitía la reestructuración de los contenidos en función del área o temática de interés del usuario. En aquel entonces observamos la potencialidad de la herramienta, cuyas funcionalidades más poderosas tuvieron que ser limitadas en favor de un más fácil manejo al no disponerse de tiempo para formación.

Aquel sistema hacía uso ya de una categorización de contenidos basada en etiquetas que tan de moda se ha puesto últimamente con la web 2.0. Ello permitía la reestructuración de la documentación de forma sencilla y rápida para presentarla desde la perspectiva de interés del usuario en cada momento.

El sistema de etiquetado surgió en las investigaciones del LITI como una necesidad para categorizar contenidos en los entornos de gestión de conocimiento desarrollados por el laboratorio, y su utilidad principal estaba vinculada al ciclo de generación de nuevo conocimiento al permitir reestructurar el repositorio por un número ilimitado de relaciones. Así, el análisis bajo nuevos puntos de vista podía llevarse a cabo con la simple introducción de etiquetas que reflejaran suficientemente cada nueva perspectiva.

La evolución de los sistemas y el planteamiento de herramientas rápidas de búsqueda permitieron descubrir la enorme potencialidad que tenía el procedimiento de etiquetado. Bastaba con identificar los puntos de vista más comunes y sus etiquetas asociadas para obtener auténticos buscadores rápidos y precisos. Si además se consigue una 


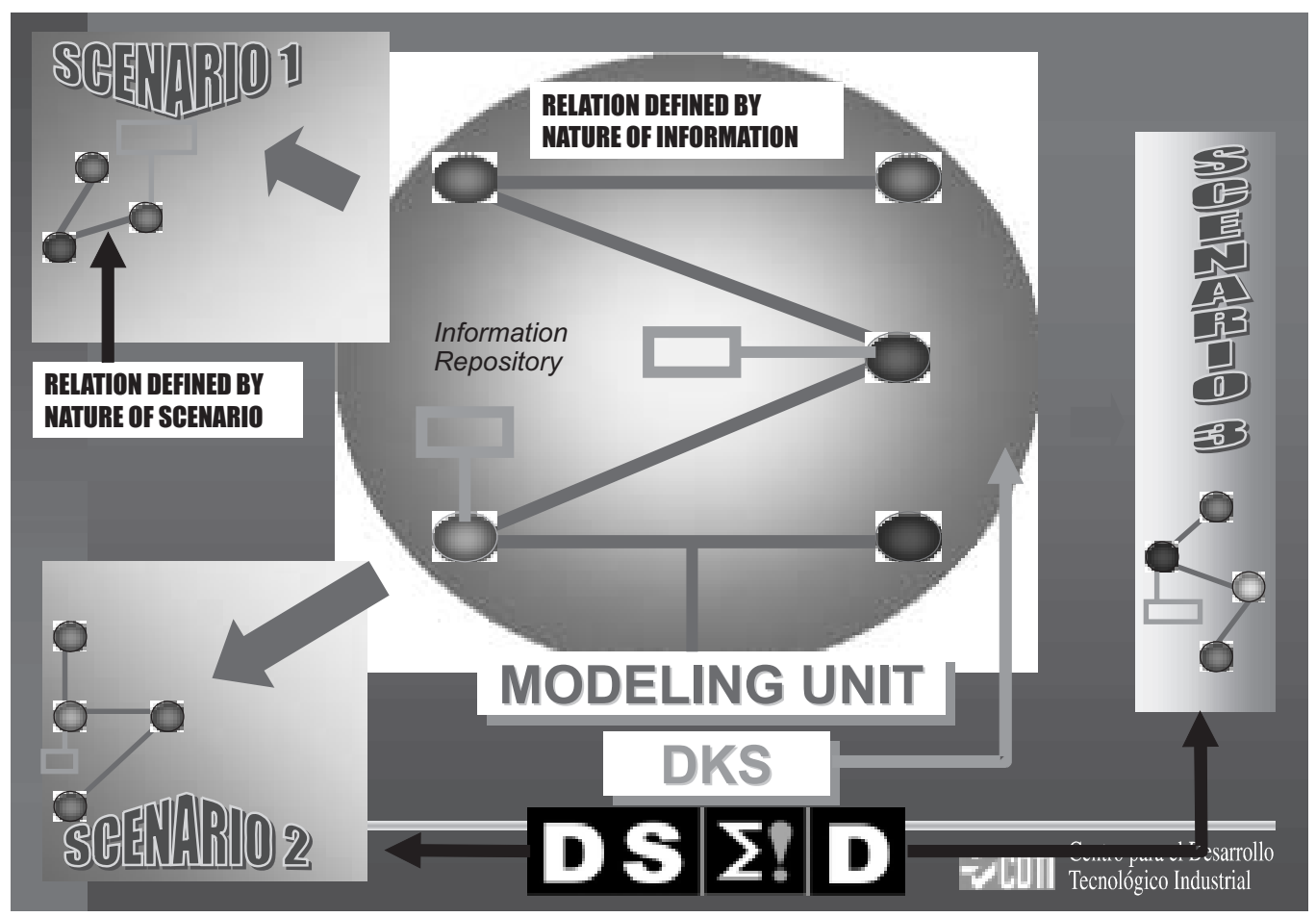

Figura 1. Esquema de funcionamiento de la herramienta diseñada por el LITI para la distribución de la documentación oficial de la última presidencia española del programa Eureka en el año 2000.

categorización semiautomática se habrá añadido entonces la importante componente de bajo coste al buscador.

La captura automática de información ha avanzado mucho en los últimos años. No obstante, muy pocos procesos automáticos son capaces de realizar una categorización completa. Por ello, desde el departamento de promoción de la innovación al analizar y definir los procesos de captura y categorización utilizamos la inestimable participación de proveedores que conocen perfectamente la estructura y el formato de los contenidos a capturar y les trasladamos nuestro punto de vista como usuarios avanzados de ese potencial buscador. Los primeros análisis sobre las capturas automáticas nos revelaron la necesidad de incorporar una pequeña y ligera tarea de categorización manual que mejoraba sensiblemente el resultado final y garantizaba una mínima supervisión. Esto suponía un pequeño coste de mantenimiento de la herramienta pero a la vez supervisaba ligeramente el trabajo y confirmaba la validez de los contenidos.
De la manera descrita fue como nos enfrentamos a una de las carencias detectadas en varios estudios ${ }^{1}$ sobre sistemas usados por los trabajadores del conocimiento: necesidad de una mejor clasificación del contenido. El sistema de categorización que surge del uso de etiquetas se llama "folksonomía" (un sistema de categorización en régimen de colaboración elaborada a lo largo del tiempo por las personas). A veces se puede interpretar la folksonomía como algo contrario a la taxonomía, donde el esquema de categorización viene determinado por un experto. Las principales ventajas de las folksonomías es que reflejan las estructuras de información y las relaciones de información que utilizan las personas en la práctica, en lugar de estructuras y relaciones planificadas de antemano e impuestas a los usuarios.

Como usuarios avanzados en la búsqueda y análisis de ayudas públicas establecimos una folksonomía a través de etiquetas que permitía estructurar las ayudas desde nuestro uso interesado y ofrecer un panorama personali- 
zado del repositorio. Si esa estructuración la ofreciamos en abierto en el buscador estábamos convirtiendo nuestro sistema de etiquetado en una taxonomía para el proceso de búsqueda. Resolvíamos así las dificultades asociadas a la categorización del repositorio y el sistema quedaba capacitado para poder cambiar la estructuración de contenidos y adecuarla a nuevos puntos de vista o usos interesados que descubriéramos en el futuro. Se había alcanzado así un procedimiento de búsqueda flexible y adaptable.

Una vez superada la dificultad de la clasificación, nos enfrentamos al siguiente reto: la correcta distribución y transmisión de la información. Cuando desde el departamento de promoción de la innovación nos planteábamos poner en marcha un sistema que permitiera a las empresas y emprendedores acceder a la totalidad de las ayudas públicas a la $\mathrm{I}+\mathrm{D}+\mathrm{i}$ éramos conscientes de la dificultad que implicaba ofrecer un elevado conjunto de conocimientos que debian de poderse transmitir desde una plataforma electrónica.

En el departamento de promoción de la innovación, y en el conjunto del Centro para el desarrollo tecnológico industrial, estamos familiarizados con la transferencia de conocimientos desde la perspectiva de la tecnología como conocimiento más usado y estudiado en nuestra actividad diaria. Sin embargo, como tal conocimiento, las conclusiones sobre su transferibilidad pueden aplicarse a cualquier ámbito o contexto puesto que el análisis se basa en atributos o dimensiones básicas del conocimiento.

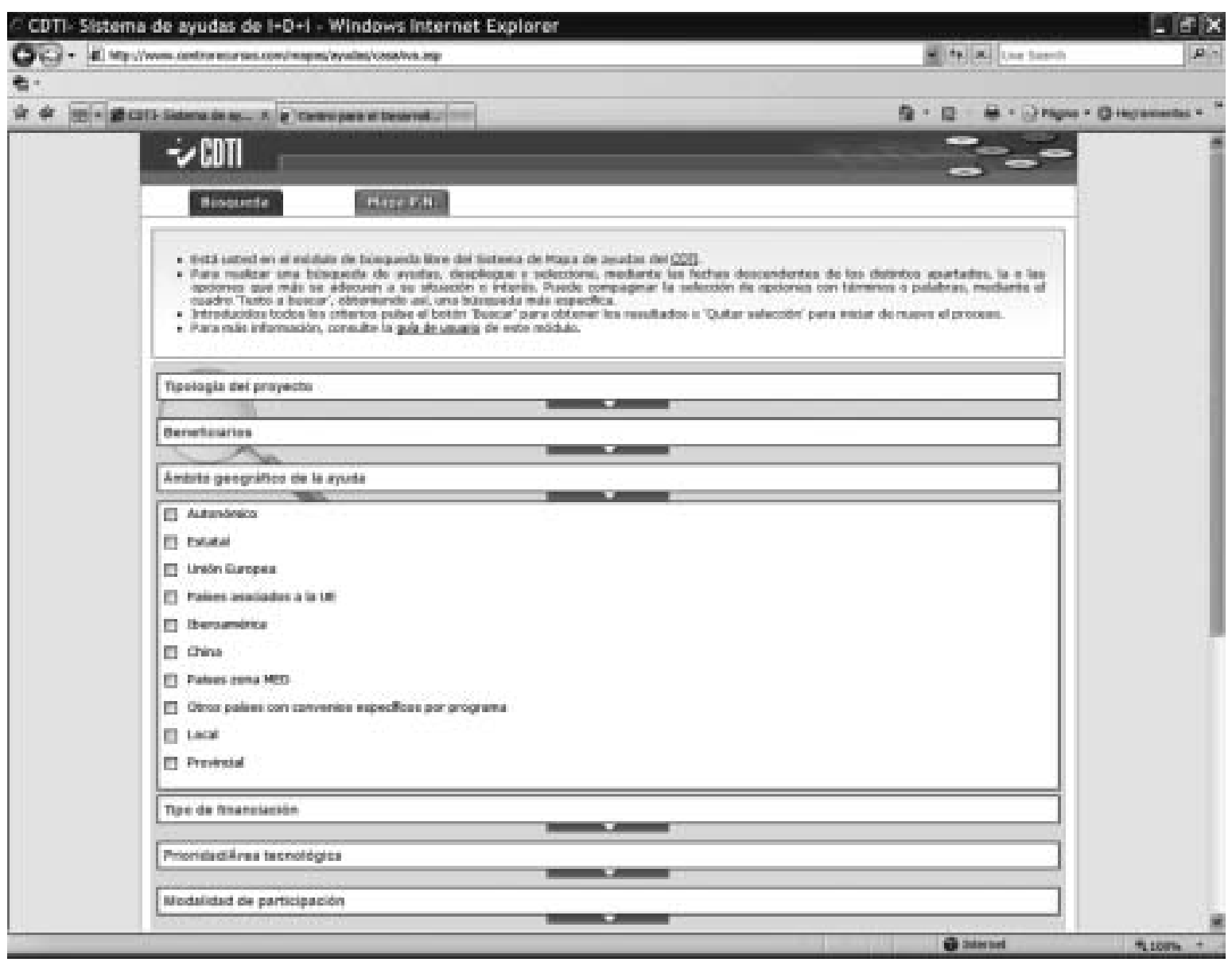

Figura 2. El buscador permite realizar las búsquedas utilizando etiquetas predefinidas que permiten visualizar los resultados desde multitud de puntos de vista. En un futuro se contempla la posibilidad de que grupos específicos de usuarios puedan definir sus propias etiquetas. 
Así, las posibilidades de que un determinado conocimiento tecnológico pueda ser transferido voluntariamente 0 imitado y la velocidad de su difusión van a depender de: (1) el nivel de formalización, (2) la capacidad para ser enseñado, (3) la posibilidad de que sea observable, (4) el grado de complejidad y (5) el grado de dependencia de otros conocimientos. Estas características del conocimiento son las que dificultan la identificación de las tecnologías relevantes y originan las imperfecciones en la transferencia de conocimientos tecnológicos (Nieto Antolín, 2001²).

El grado en que los conocimientos puedan ser codificados, enseñados y observados va a influir en las posibilidades de que sean identificados y transmitidos. Si el conocimiento es explícito, se puede enseñar con facilidad y es observable, su identificación por parte de terceros será inmediata. Los ejemplos de este tipo de conocimientos son enormemente variados; no obstante pueden agruparse en cuatro categorías básicas (Badaracco, 19913):

1. El conocimiento contenido en textos, diseños y procedimientos. Son desarrollos teóricos formalizados, cálculos, fórmulas, memorias de ingeniería, planos, especificaciones técnicas, y otros documentos.

2. El conocimiento contenido en las propias máquinas. Todas las máquinas son en cierto modo un depósito de "conocimiento congelado", que puede extraerse mediante la ingeniería inversa. También, ciertas máquinas poseen conocimiento en la medida que saben cómo desempeñar ciertas tareas como los ordenadores con sistemas expertos, equipos de análisis y diagnóstico, etcétera.

3. El conocimiento contenido en ciertos materiales: Productos químicos y farmacéuticos, metales de aleaciones especiales, nuevos materiales de caracteristicas avanzadas, polímeros, plásticos, etc.

4. El conocimiento contenido en la mente de los individuos. No todo el conocimiento que poseen los individuos es de carácter tácito. También, las personas poseen conocimientos explícitos que pueden ser observados y enseñados con facilidad. Prueba de ello es que los ingenieros y directivos de las empresas son capaces de transmitir parte de sus conocimientos a otros miembros de la organización mediante cursos de formación convencionales. Los potenciales imitadores, pueden identificar esos conocimientos del personal de otra empresa sin más que observar sus compor- tamientos y analizar las decisiones que adoptan. En caso extremo, pueden adquirir estos conocimientos contratando a ese personal.

Estos tipos de conocimientos por ser explícitos, enseñables y observables no van a tener especiales dificultades para ser identificados y van a poder adquirirse e imitarse fácilmente. Conforme a esto, cuando los conocimientos tengan estas características van a difundirse y transmitirse con rapidez.

Por el contrario, si los conocimientos son tácitos, difíciles de enseñar y observar van a tener una menor probabilidad de ser identificados. Estos conocimientos, residen fundamentalmente en las relaciones especializadas entre los individuos y los grupos y en las rutinas, actitudes y formas de tomar decisiones que caracterizan los contactos entre ellos. Estos conocimientos, surgen a partir de la experiencia acumulada en la mejora de las operaciones cotidianas como resultado de un largo proceso de aprendizaje. Están incrustados en las rutinas de las organizaciones y es muy difícil su identificación.

Por otra parte, el grado de complejidad y grado de dependencia que tenga el conocimiento reducen las posibilidades de que pueda ser usado eficazmente. En general, cuanto mayor sea la complejidad del conocimiento tecnológico y mayor su grado de dependencia de otros conocimientos más difícil y lenta será su transferencia.

Todo conocimiento complejo surge de la combinación de un conjunto heterogéneo de otros conocimientos. Cada uno de estos conocimientos, a su vez, puede ser el resultado de la integración de otra serie de conocimientos complejos y así sucesivamente. Es obvio que cuanto más complejo sea un paquete de conocimiento, más dificultad se tiene para identificar y reproducir todo el conjunto de conocimientos individuales que lo forman.

Con independencia de su complejidad, un determinado conocimiento tecnológico puede ser dependiente, y estar integrado, en un sistema de conocimientos. Es decir, para su plena utilización puede ser necesario el empleo de una serie de conocimientos complementarios (otras tecnologías, experiencia anterior, personal especializado,...). En algunos casos, la disponibilidad de recursos complementarios, es imprescindible para asegurar el éxito del 
proceso de innovación. Obviamente una organización o individuo tendrá dificultades para utilizar eficazmente un determinado conocimiento sin poseer los conocimientos complementarios adecuados. Incluso, su utilización sin esos conocimientos complementarios podrá ser perjudicial. Por ello, la transferencia de un conocimiento va a estar afectada por su grado de dependencia respecto a otros conocimientos. Cuanto mayor sea el número de relaciones de dependencia de un conocimiento respecto a otros más lenta y difícil va a ser su transferencia.

En resumen, si el conocimiento es tácito, no enseñable y no observable, será difícil identificarlo. Por otro lado, si el conocimiento es complejo y dependiente, será difícil conocer los vínculos que lo ligan a otros conocimientos, lo que aumentará la dificultad de uso. Los problemas de identificación y uso del conocimiento van a limitar su transferencia.

Por tanto, nuestro sistema de búsqueda e información de ayudas públicas a la $\mathrm{I}+\mathrm{D}+\mathrm{i}$ tenía que ofrecer un conocimiento lo más explícito, enseñable y observable posible, además tenía que contemplar la posibilidad de acceder a los conocimientos previos de los cuales dependian los contenidos que se obtuvieran en las búsquedas. En nuestro caso, dado que el conocimiento ofrecido es aquel vinculado a una convocatoria pública de ayuda, los conocimientos previos necesarios para su asimilación venían recogidos

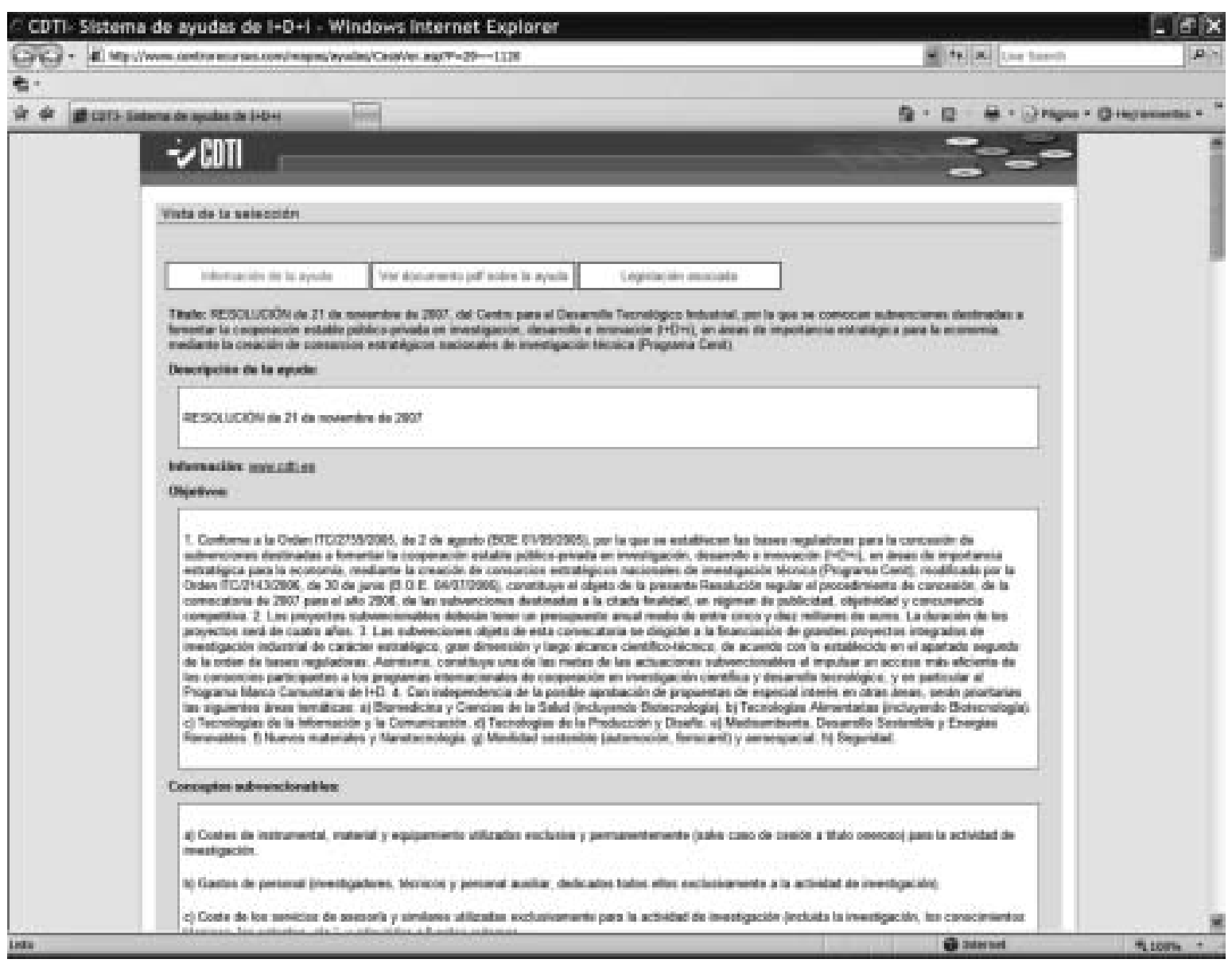

Figura 3. Además de la ficha informativa de la ayuda, el buscador proporciona la publicación del boletín oficial y toda la legislación asociada a esa ayuda en formato pdf con indexación del contenido. 
en su mayoría en la orden u órdenes de bases previa que se hubiera publicado y afecten a ese tipo de ayudas. El sistema visualiza el resultado de cada búsqueda con todos los documentos oficiales publicados y relacionados con la ayuda objeto de la búsqueda cumpliendo así el requisito imprescindible para la transferibilidad.

Además, a través de un pequeño trabajo adicional, y gracias al buen desempeño que tenía el proveedor que capturaba automáticamente los textos de los distintos boletines oficiales, se incorporó una indexación de los textos oficiales lo que permitía una fácil detección y observación de los contenidos. Se cumplía así el resto de requisitos para conseguir una buena transferencia de conocimiento a través del sistema, el conocimiento ofrecido era enseñable y fácilmente observable.

En conclusión, los pasos anteriores describen los hitos principales por los que ha discurrido el desarrollo del "Mapa electrónico de ayudas a la I+D+i" del Centro para el Desarrollo Tecnológico Industrial (CDTI) que permite acceder de forma fácil y accesible a información actualizada sobre todas las ayudas públicas que las administraciones (local, autonómica, estatal y europea) ponen a disposición de empresas, emprendedores y centros de investigación para fomentar la realización de actividades de investigación, desarrollo e innovación (I+D+i) empresarial. La actualización de la información se realiza de forma diaria a través de un proceso de captura automática en el conjunto de boletines oficiales del conjunto de administraciones antes mencionadas.

Este mapa de ayudas forma parte de la batería de herramientas de soporte de la Red de Puntos de Información sobre I+D+i (PIDi), una iniciativa lanzada en julio de 2006 cuyo objetivo es ofrecer servicios de información y asesoramiento a empresas y emprendedores sobre las ayudas públicas a la I+D+i. La utilización de esta herramienta, accesible en abierto a través de la página web del CDTI -www.cdti.es-, es completamente gratuita, y permite acceder a la información por múltiples criterios de selección: áreas tecnológicas de actividad de la empresa, tamaño de la empresa requerido para su participación, localización geográfica en la que se desarrollará el proyecto, sector de actividad, tipo de financiación que se precisa o modalidad de participación individual o cooperativa, entre otros criterios.
El mapa, totalmente automatizado y con una actualización diaria, ha permitido informar ya sobre más de un millar de ayudas a la $\mathrm{I}+\mathrm{D}+\mathrm{i}$ con un gran nivel de detalle sobre los requisitos de acceso, sobre sus posibles beneficiarios, sobre los importes destinados a cada ayuda y los plazos de presentación, llegando a incluir un índice con el texto completo de la publicación oficial de la ayuda para su más fácil comprensión. El servicio ha recibido ya más de 31.000 visitas correspondientes a más de 9.800 usuarios. Pero todavía más importante que los datos son los aspectos cualitativos que incorpora este mapa, al ofrecer la actuación de las distintas administraciones de una manera conjunta lo que aporta coherencia y eficiencia a la acción de apoyo público hacia las iniciativas innovadoras y permite, además, al potencial usuario de ese apoyo descubrir las mejores oportunidades o actuaciones desde su punto de vista.

Sin duda, este mapa, disponible para toda la comunidad investigadora española, no hubiera sido posible sin la colaboración tecnológica del Laboratorio de Innovación en Tecnologías de la Información de la Universidad Politécnica de Madrid que aportó y sigue aportando su saber en el área de herramientas dedicadas a la gestión de conocimiento que soportan este Mapa de ayudas permitiendo la carga, clasificación, organización y validación de ayudas de forma distribuida y cooperativa a través de folksonomías, al igual que el software social. Esto último supone una innovación que permite dar respuesta a distintas situaciones de búsqueda, por ejemplo buscar especificando el tipo de empresa o el proyecto que desea realizar. Tradicionalmente los buscadores parten de la idea de que el usuario conoce la naturaleza y ciertas particularidades de lo que está buscando; con esta innovación, además, se pueden realizar búsquedas de alta precisión condicionadas por las características de la acción que se quiere desarrollar con el contenido buscado.

Destacan también otras innovaciones del sistema, relacionadas con la gestión del conocimiento, como la posibilidad de agrupar los resultados de búsquedas (de esta forma los usuarios tendrán organizadas las ayudas por distintos ámbitos: europeos, estatales, regionales,..) o generar informes personalizados seleccionando la totalidad o parte de los resultados de la búsqueda (dichos informes son editables, accesibles desde Internet y gestionados de forma independiente al buscador...). 


\section{NOTAS}

Recibido: 18 de marzo de 2009

Aceptado: 16 de abril de 2009
1 Estudio de Forrester Research mencionado en "Empresa 2.0: el nacimiento de la colaboración emergente", Andrew P. McAfee, Harvard Deusto Business Review, 2007.
2 Mariano Nieto Antolín (2001), Bases para el estudio del proceso de innovación tecnológica en la empresa, Universidad de León.

3 Badaracco Jr., J. L. (1991), The Knowledge link: how firms compete through strategic alliances, McGrawHill, New York. 